\title{
Tetraeder Geometry
}

\author{
Dipl.-Phys. Ulrich Winter \\ U.P.D.I., 53757 St. Augustin, Ahrstr. 1, Germany \\ Ulrich.Winter.2015@NeuronX.de
}

\section{Abstract}

In the same way every circle is corresponding to an equal sided triangle the 3 -sphere is corresponding to an inserted tetraeder. The latiude of the corners of the tetraeder on the 3 -sphere is calculated here giving the result arcsin(1/3), while $\arcsin (1 / 2)$ is derived for the 2-dimensional sphere.

\section{Keywords}

Geometry, Tetraeder, Dimension

\section{Introduction}

In the same way every circle is corresponding to an equal sided triangle the 3 -sphere is corresponding to an inserted tetraeder. The latiude $\square$ of the corners of the tetraeder on the 3-sphere is calculated here.

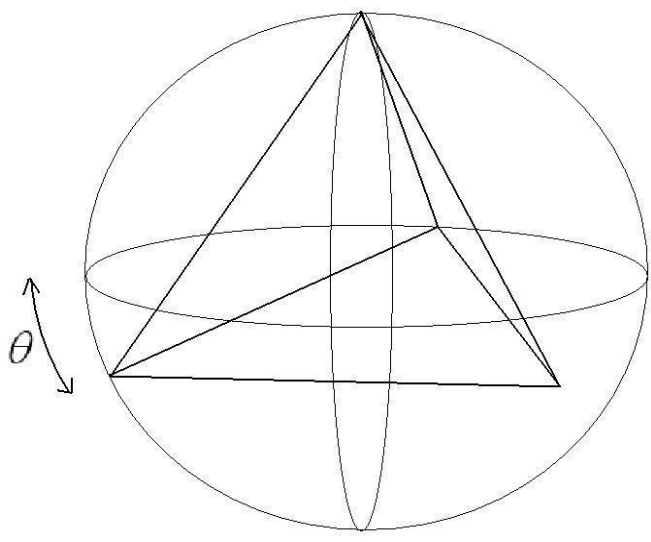

Fig.1: Tetraeder in 3-sphere

\section{Method}

The rules found by Pythagoras and angular relations are the only methods necessary to calculate the angle. First we have to consider the triangle forming one side of the tetraeder.

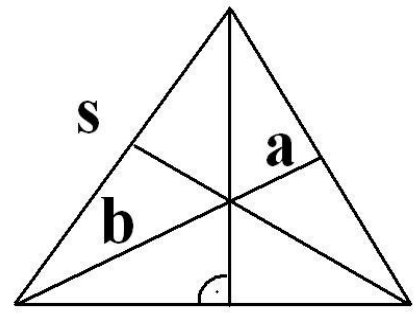

Fig.2: Triangle on one side of the tetraeder

The first relation we need is the Pythagorean theorem[1], p.244, in two cases:

$$
\left(\frac{s}{2}\right)^{2}+h_{\text {Triangle }}^{2}=s^{2}
$$


These two equations resolve to

$$
a^{2}+\left(\frac{s}{2}\right)^{2}=b^{2}
$$

$$
a^{2}+\frac{1}{2} a b-\frac{1}{2} b^{2}=0
$$

with the solution

$$
a=\frac{1}{2} b
$$

$(1)=>$

$$
h_{\text {Triangle }}=\frac{1}{2} \sqrt{3} \mathrm{~s}
$$

gives:

$$
\begin{aligned}
& \sin 30^{\circ}=\frac{a}{b}=\frac{1}{2} \\
& a+b=h_{\text {Triangle }}
\end{aligned}
$$

(5), (6) and (7) gives us

$$
\begin{aligned}
& a+b=\frac{b}{2}+b=\frac{3}{2} b=\frac{1}{2} \sqrt{3} s \\
& b=k s
\end{aligned}
$$

defining

$$
k \stackrel{\text { def }}{=} \frac{1}{\sqrt{3}}
$$

Let us consider now the triangle in the midth of the tetraeder.

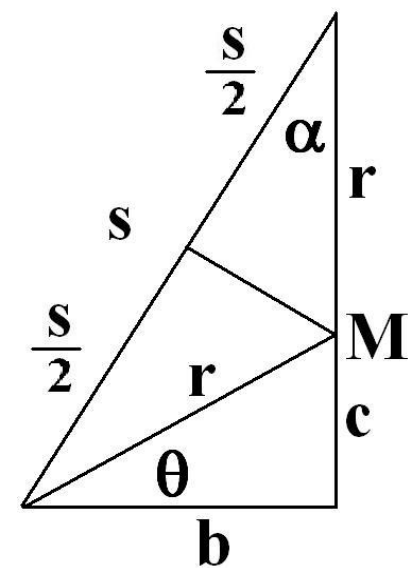

Fig. 3 Triangle in the midth of the tetraeder 


$$
\sin \theta=\frac{c}{r}
$$

$=>$

$$
\sin \theta=\frac{h-r}{r}
$$

$=>$

$$
\sin \theta=\frac{\sqrt{s^{2}-b^{2}}-r}{r}
$$

If we apply the law of cosines [1], p.253, on the triangle in Fig.3 we get

$$
\cos \alpha=\frac{\left(\frac{s}{2}\right)}{r}
$$

and so for s

$$
s=2 r \cos \alpha
$$

Inserting (8) into (12) we get

$$
\sin \theta=\frac{\sqrt{s^{2}-s^{2} k^{2}}-r}{r}
$$

$=>$

$$
\begin{aligned}
& \sin \theta=\frac{2 r \cos \alpha \sqrt{1-k^{2}}-r}{r} \\
& \sin \theta=2 \cos \alpha \sqrt{1-k^{2}}-1
\end{aligned}
$$

If we apply again the law of cosines [1], p.253, on the complete triangle in Fig.3 and in the next step the Pythagorean theorem[1], p.244, and insert (8) in the last step we get

$$
\begin{gathered}
\cos \alpha=\frac{c+r}{s} \\
=\frac{\sqrt{s^{2}-b^{2}}}{s}=\frac{\sqrt{s^{2}-k^{2} s^{2}}}{s}
\end{gathered}
$$

which evaluates to

$$
\begin{gathered}
\cos \alpha=\sqrt{1-k^{2}} \text { Using this (17) becomes } \\
\sin \theta=2 \sqrt{1-k^{2}} \sqrt{1-k^{2}}-1
\end{gathered}
$$




\section{Result}

By inserting the definition for $k$ (9) into equation (19) we get the final result.

$$
\sin \theta=\frac{1}{3}
$$

or

$$
\theta=\arcsin \frac{1}{3}
$$

The numerical value for the latitude of equation (21) is

$$
\theta=19.47122063^{\circ}
$$

or

$$
\theta=19^{\circ} 28^{\prime} 16^{\prime \prime}
$$

\section{Conclusion}

This calculation for the latitude of a corner of an equal sided triangle in a two dimensional sphere and a tetraeder inside a three dimensional sphere resulted in a simple formula containing the dimension of the considered geometry in the denominator.

The two dimensional latitude we got from Fig. 2 and (6) as

$$
\begin{aligned}
& \theta_{2-\operatorname{dim}}=\arcsin \frac{1}{2} \\
& \theta_{3-\operatorname{dim}}=\arcsin \frac{1}{3}
\end{aligned}
$$

or generalized

$$
\text { Latitude }_{n-\operatorname{dim}}=\arcsin \frac{1}{n}
$$

which is at least valid for the equal sided bodies in the 2-dimensional-sphere and the

3-dimensional-sphere as calculated here and may be valid also for higher dimensional spheres or fractal dimensions, if it proves to be a general law.

If we consider the rule of construction and extend it to the 1-dimensional and the 4-dimensional-sphere we get: 


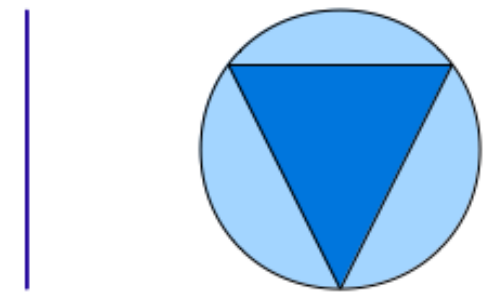

\section{dimension $=1$ $\arcsin (1 / 1)$} $90^{\circ}$

dimension=2
$\arcsin (1 / 2)$
$30^{\circ}$
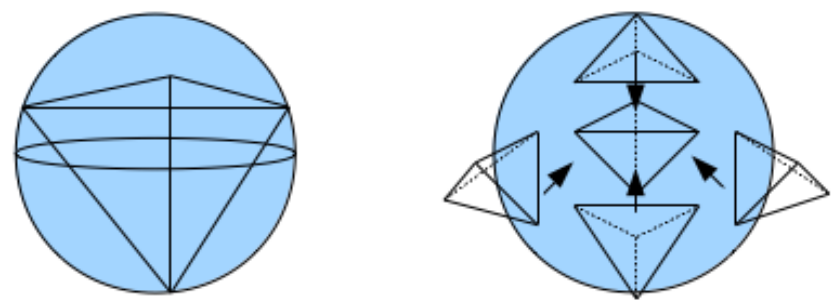

Fig.4:

dimension=3
$\arcsin (1 / 3)$

$19.47^{\circ}$

\section{dimension=4 $\arcsin (1 / 4) ?$}

$14.47^{\circ} ?$

General Construction Rule for dimension n:

1. draw the n-dimensional sphere

(i.e. draw 2 points at distance $r$ in 1-dim, a circle in 2-dim, a 3-sphere in 3-dim )

2. into the sphere draw $n+1$ connected $n-1$ dimensional objects and connect the $n-2$ dim edges

The "n-1 dimensional object" is a point in 1-dim, a line in 2-dim, an area in 3-dim, a tetraeder in 4-dim space. The fourth dimension is the time, so that the fourth figure shows a 3-sphere in time.

\section{Discussion}

The construction rule is a little bit hard to imagine for the 4-dimensional case. The rule not only demands the four tetraeder connecting side by side to the fifth one. Also each of the other sides of the tetraeders have to be connected to a matching side of another tetraeder forming a closed connected 3-dimensional manifold.

The property "closed" implies, that there is no way to leave the volume by going in any direction.

The property "connected" implies, that there are no borders or holes in this volume.

The limited size of the volume is just five times the volume of one tetraeder.

This model may be compared to the known cosmological model having a diameter of $8.8 \times 10^{26} \mathrm{~m}$ [6] and a limited volume of $4 \times 10^{80} \mathrm{~m}^{3}$ [5].

In literature, p.e. Wikipedia [2], the four dimensional case is constructed with inwards pointing tetraeder. In this case the volume is harder to see, but the connection of the sides of the tetraeder is established directly. The four dimensional object is called in literature a "4-polytope" [2] or a "Polychoron" [3].

\section{References}

It was the own idea of the author of this paper to do all the calculations listed here.

The results (22) and (23) are not listed in any textbook or publication so far, which motivated the author to publish these here in the hope it will be usefull for further calculations.

The law of Pythagoras and the other triangular relationships are listed in many textbooks of Geometry, for instance the one listed here [1].

1. I. N. Bronstein, K.A. Semendjajew, Taschenbuch der Mathematik, Verlag Harri Deutsch, Thun und Frankfurt (Main), 20. Auflage, Germany, (1981)

2. Wikipedia, 4-Polytype, https://en.wikipedia.org/wiki/4-polytope

3. Wolfram MathWorld, Regular Polychoron, http://mathworld.wolfram.com/RegularPolychoron.html

4. Wikipedia, Observable Universe, https://en.wikipedia.org/wiki/Observable_universe

5. Wolfram Alpha, Volume Universe, ttp://www.wolframalpha.com/input/?i=volume+universe

6. Itzhak Bars; John Terning (November 2009). Extra Dimensions in Space and Time. Springer. pp. 27, ISBN 978-0387-77637-8. Retrieved 2011-05-01. 


\section{Dipl.-Phys. Ulrich Winter biography}

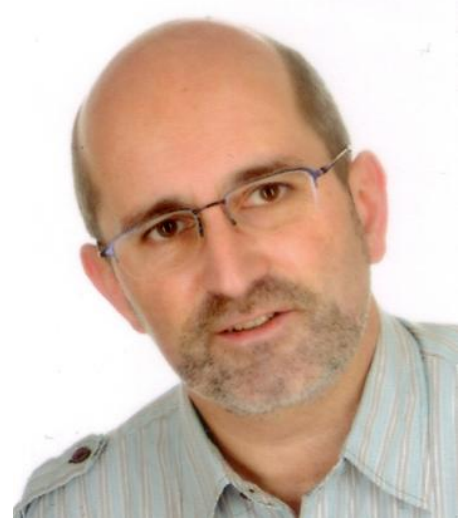

2010 Presentation of "The Two Ways of Contact" at the N.A.S.A. Contact Conference, St. Jose, California, USA

2009 Presentation of "N.I.N.A.", the Neural Integrated Network Architecture, at the Maritim, Königswinter, Germany

2008 Presentation of the visualization of the new solutions for the field equations of Einstein's General Relativity Theory

at the Uni-Phi Conference on Hawai'i

2004 Analysis work and start of the calculations on Einstein's Theory of Gravity

1991 - 2017 working as a system analyst and software developer in several industry projects for

Toyota, Japan, TechMahindra, India and T-Mobile, Germany

1991 Physics Diplom (University Bonn, Germany)

1981 Highschool Absolvent in Geldern, Germany

1962 born on the lovely countryside between the two rivers Rhine, Germany, and Maas, Netherlands 